\title{
The Effect of PEG Crystallization on the Morphology of PEG/Peptide Block Copolymers Containing Amyloid $\beta$-Peptide Fragments
}

\author{
Marta J. Krysmann, Sérgio S. Funari, Elisabetta Canetta, Ian W. Hamley*
}

Ordered nanostructures are observed in the melt and solid state for a series of three peptide/ PEG conjugates containing fragments of amyloid $\beta$-peptides. These are conjugated to PEG with $\bar{M}_{\mathrm{n}}=3300 \mathrm{~g} \cdot \mathrm{mol}^{-1}$ and a melting temperature $T_{\mathrm{m}}=45-50^{\circ} \mathrm{C}$. The morphology at room temperature is examined by AFM and POM. This shows spherulite formation for the weakly fibrillizing KLVFF-PEG sample but fibril formation for FFKLVFF-PEG. The fibrillization tendency of the latter is enhanced by multiple phenylalanine residues. Simultaneous SAXS and WAXS was used to investigate the morphology as a function of temperature. The secondary structure is probed by FTIR.

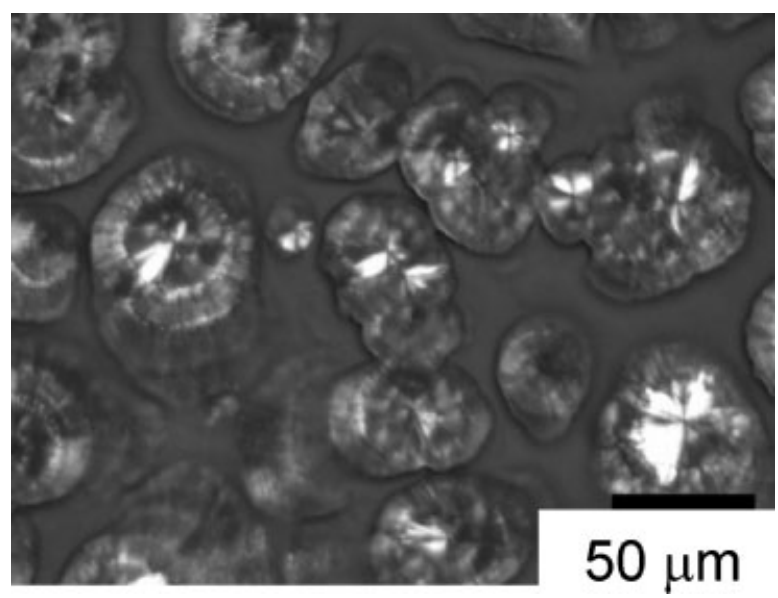

\section{Introduction}

The conjugation of peptides to synthetic polymers offers the potential to create novel materials with hybrid properties. ${ }^{[1-6]}$ The self-assembly of the peptide can be influenced by the synthetic polymer, resulting in distinct tertiary structures. From another viewpoint, peptide

M. J. Krysmann, I. W. Hamley

Dept of Chemistry, University of Reading, Reading RG6 6AD, UK E-mail: i.w.hamley@reading.ac.uk

S. S. Funari

HASYLAB, Notkestrasse 85, Hamburg D-22603, Hamburg,

Germany

E. Canetta

Department of Physics, University of Surrey, Guildford, Surrey

$\mathrm{GU}_{2} 7 \mathrm{XH}$, UK

I. W. Hamley

Diamond Light Source, Chilton, Didcot, OX11 ODE, UK functionalities can be attached to synthetic polymers conferring novel ligands that can self-assemble or interact with biomaterials in a targeted fashion.

There is great interest in amyloid formation due to its probable role in diseases including Alzheimer's, bovine spongiform encephalopathy etc. ${ }^{[6-11]}$ Amyloids are deposits of fibrillar aggregates that form when peptides misfold from their native structure and adopt a cross-beta sheet configuration, with beta strands perpendicular to the long fibril axis. The amyloid beta peptide $(\mathrm{A} \beta)$ undergoes amyloidosis in humans. The peptide is enzymatically cleaved from the amyloid precursor protein to produce fragments with 39-43 residues, especially $\mathrm{A} \beta(1-40)$ and $\mathrm{A} \beta(1-42) \cdot{ }^{[6]}$ We are presently investigating the selfassembly of fragments of the peptide KLVFF [A $\beta(16--20)]$, hydrophobically-modified versions of this sequence, and additionally of PEGylated peptides. PEGylation is of interest because it confers solubility on hydrophobic peptides, PEG being biocompatible, providing steric stabilization and 
conferring enhanced blood circulation time. The peptide copolymers may thus ultimately have applications as therapeutic agents in the treatment of amyloid diseases. The focus of the present paper is on self-assembly in the solid state, however. We investigate the interplay between peptide secondary-structure formation and microphase separation in the melt and in the solid state where PEG crystallizes.

There have been relatively few studies to date concerning the interplay between PEG crystallization and peptide secondary-structure formation. Prior work on the solidstate structure of PEG-peptide block copolymers has been reviewed. ${ }^{[12]}$ Regarding PEG-peptide copolymers with non-synthetic peptide sequences, Rösler et al. investigated the effect of PEG crystallization on the solid-state selfassembly of PEG/peptide diblocks and PEG/peptide/PEG triblocks containing peptides with 18 residues from a biomimetic "switch" sequence. ${ }^{[13]}$ The $\beta$-strand peptide secondary structure was retained even when PEG crystallized. Smeenk and coworkers have investigated fibril formation by a series of PEG/peptide/PEG block copolymers in which the central PEG domain was based on the $\left[(\mathrm{AG})_{3} \mathrm{EG}\right]_{10}$ sequence. ${ }^{[14]}$ Peptide $\beta$-sheet formation was unaffected by conjugation to PEG. Only for highmolar-mass PEG $\left(5000 \mathrm{~g} \cdot \mathrm{mol}^{-1}\right)$ could an effect on fibril formation be observed, in particular a reduction in fibril length. Burkoth and coworkers previously investigated fibril formation of $A \beta(10-35)-P E G 3000$ in aqueous solution via transmission electron microscopy (TEM) and smallangle neutron scattering (SANS). ${ }^{[15-17]}$ SANS indicated the formation of fibrils with a peptidic core and a PEG corona. ${ }^{[16,17]}$ The corona dimensions were consistent with the radius of gyration of PEG3000 and the core radius was slightly smaller than that of a fully extended $\beta$-strand and decreased with decreasing $\mathrm{pH}^{[17]}$

In the present paper, we investigate self-assembly in the melt and solid state of a series of PEG-peptide copolymers. Three copolymers are studied: KLVFF-PEG, AAKLVFF-PEG and FFKLVFF-PEG. The peptide sequences are based on KLVFF, sequence $\mathrm{A} \beta(16-20)$ from the $\mathrm{A} \beta$ peptide and hydrophobically-modified variants of this. The inclusion of additional hydrophobic units enhances the tendency for fibrillization (in an appropriate solvent) which is rather weak in the KLVFF homo-polypeptide. ${ }^{[18-20]}$ The peptides are conjugated with PEG with $\bar{M}_{\mathrm{n}}=3300 \mathrm{~g} \cdot \mathrm{mol}^{-1}$.

\section{Experimental Part}

\section{Materials}

The preparation and purification of the PEGylated conjugates by solid-phase peptide-synthesis methods (using a PEG-loaded resin) is described elsewhere. ${ }^{[21]}$

\section{Small-Angle X-Ray Scattering (SAXS)}

SAXS experiments were performed on beamline A2 of HASYLAB, Hamburg, Germany. Samples in powder form were placed in $\mathrm{Al}$ foil and mounted in a heated cell. Two linear Gabriel-type detectors were used to acquire the SAXS and wide-angle X-ray scattering (WAXS) data simultaneously. The wavenumber [ $q=4 \pi \cdot(\sin \theta) / \lambda$ (scattering angle $2 \theta$, wavelength $\lambda=1.5 \AA$ ) ] scale was calibrated for SAXS using mineralized rat-tail tendon, and for WAXS using a sample of poly(ethylene terephthalate).

\section{Atomic Force Microscopy}

The AFM samples were prepared by passively absorbing aliquots of $25 \mu \mathrm{L}$ of PEG-peptide suspensions in methanol onto a freshlycleaved mica sheet. The samples were then left to dry at room temperature for a few minutes.

AFM images were obtained with an NTEGRA instrument (NT-MDT, Moscow, Russia). The samples were scanned in intermittent-contact mode by using a gold-coated silicon cantilever (NT-MDT, Moscow, Russia) with a nominal spring constant of $5.5 \mathrm{~N} \cdot \mathrm{m}^{-1}$ and a nominal resonance frequency of $150 \mathrm{~Hz}$. An ultrasharp, conical silicon tip with a typical radius of curvature of $10 \mathrm{~nm}$ was microfabricated on the cantilever. All of the AFM experiments were performed in air at room temperature, and the images were captured using a scan speed of $1.5 \mathrm{~Hz}$ with scan sizes ranging from 10 to $0.3 \mu \mathrm{m}$. The scanning parameters that control the intermittent contact conditions during the scan are the free amplitude $A_{0}$, which corresponds to the oscillation of the cantilever in air, and the setpoint amplitude $A_{\text {sp }}$ that describes the amplitude of the oscillation of the cantilever when the tip is in contact with the sample surface. All of the AFM images were captured with very-similar intermittent-contact conditions. Typically, $A_{0}$ was $25 \mathrm{nA}$ and $A_{\mathrm{sp}}$ was $20.9 \mathrm{nA}$ for all of the experiments.

The raw AFM images were deconvoluted using the NT-MDT deconvolution software (NT-MDT, Moscow, Russia) to take into account the shape of the conical tip, and then processed using the NT-MDT image processing software (NT-MDT, Moscow, Russia).

\section{Polarized Optical Microscopy (POM)}

Samples (5 wt.-\% in water) were placed onto glass microscope slides and dried. Images between crossed polarizers were obtained with an Olympus CX-41 microscope.

\section{Fourier-Transform Infrared (FTIR) Spectroscopy}

IR spectra including the amide bands were recorded on an FTIR spectrometer equipped with a deuterated triglycine sulfate (DTGS) detector. A solid film of dry PEGylated peptide was deposited on a $\mathrm{CaF}_{2}$ plate by drying a 5 wt.-\% solution. Spectra were scanned 64 times over the range of 4000 to $400 \mathrm{~cm}^{-1}$. Spectral Manager for Windows was used for data acquisition and handling.

\section{Results}

SAXS data showing reversible transitions in the morphology together with simultaneous WAXS data showing 


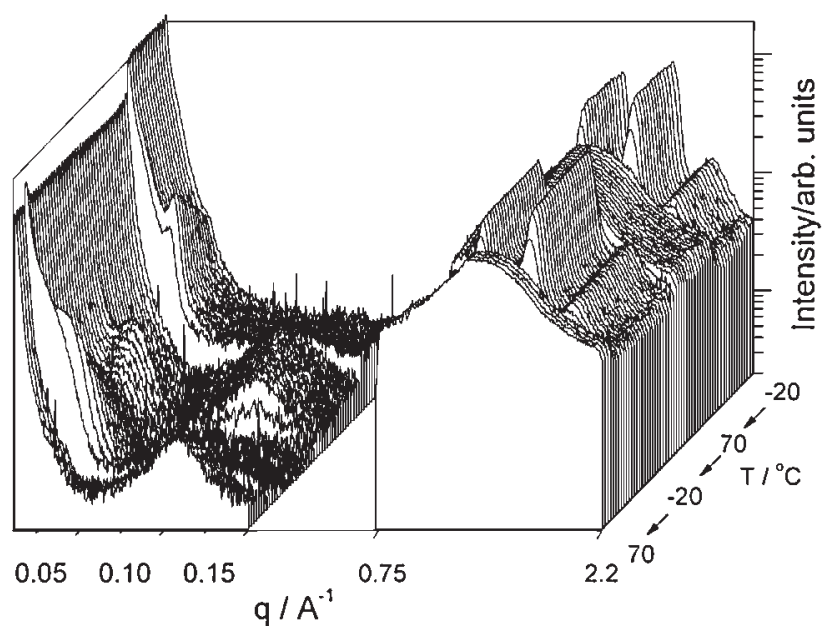

Figure 1. Temperature-dependent SAXS/WAXS data for KLVFFPEG (heating/cooling at $2{ }^{\circ} \mathrm{C} \cdot \mathrm{min}^{-1}$, holding for $5 \mathrm{~min}$ at intermediate steps at $70^{\circ} \mathrm{C}$ and $-20^{\circ} \mathrm{C}$ ).

melting and recrystallization of PEG for sample KLVFF-PEG are shown in Figure 1. Similar data were obtained for the other copolymers. The WAXS data clearly shows peaks from crystalline PEG at low temperature and a broad peak from amorphous PEG above $T_{\mathrm{m}}$. The SAXS data for the other copolymers are summarized in terms of representative profiles at $70^{\circ} \mathrm{C}$, where PEG is amorphous (Figure 2) and at $20^{\circ} \mathrm{C}$ where it is crystalline (Figure 3 ).

Focusing first on microphase separation in the melt, Figure 2 summarizes data for all three PEG/peptide conjugates at a reference temperature $T=70^{\circ} \mathrm{C}$, well above $T_{\mathrm{m}}(\mathrm{PEG})$. The presence of a peak in the SAXS data provides evidence for microphase separation in the melt for all samples. The peak is well defined for the shorter peptide, but rather broad for FFKLVFF-PEG and especially AAKLVFF-PEG.

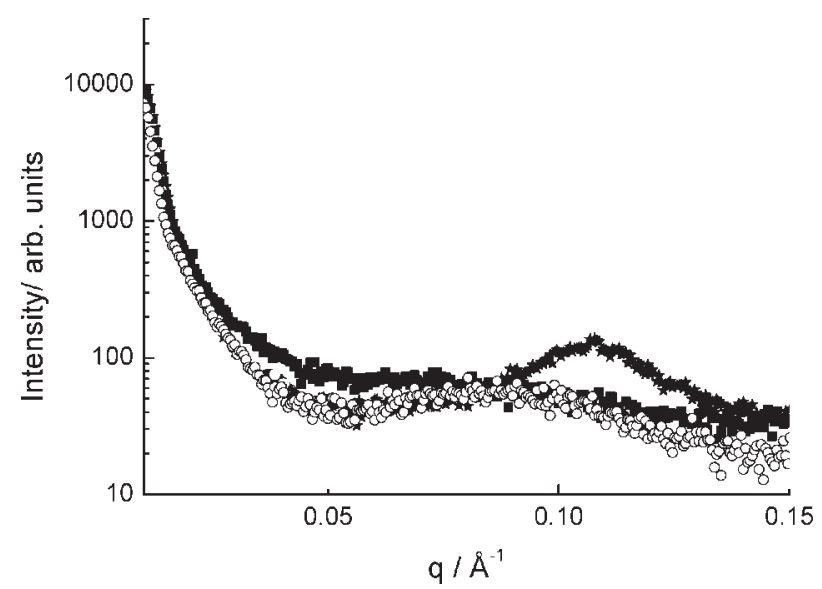

Figure 2. SAXS showing microphase-separated structures in the melt at $T=70^{\circ} \mathrm{C}$. (*) KLVFF-PEG, (ם) AAKLVFF-PEG, (o) FFKLVFF-PEG.

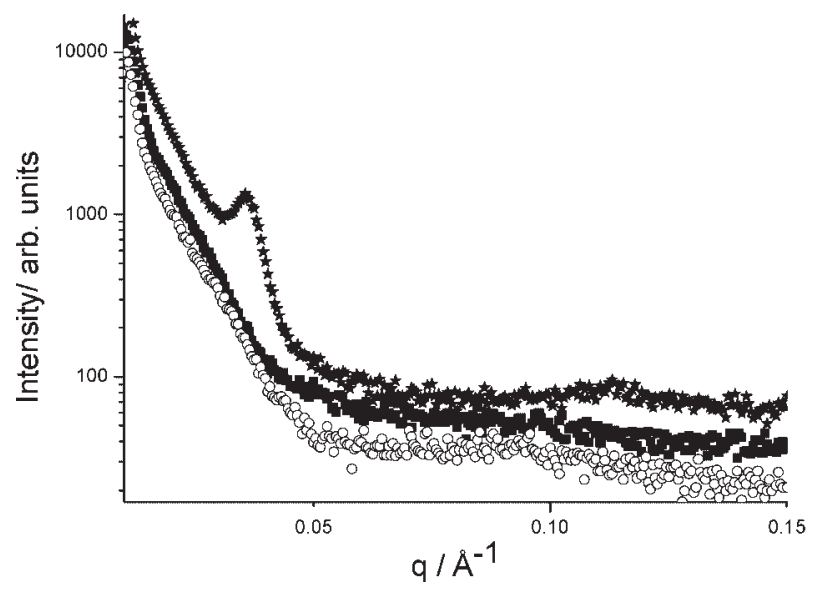

Figure 3. SAXS obtained at $T=20^{\circ} \mathrm{C}$. The results for KLVFF-PEC show Bragg peaks from PEG crystal lamellae; the results for AAKLVFF-PEG and FFKLVFF-PEG do not. (*) KLVFF-PEG,

AAKLVFF-PEG, (o) FFKLVFF-PEG.

Values of domain spacing, determined from the positions of the peak maxima, are listed in Table 1. For samples FFKLVFF-PEG and AAKLVFF-PEG the domain spacings agree very well with the model shown in Figure 4 , which comprises (in an assumed lamellar structure) opposed parallel $\beta$-strands attached to amorphous PEG. The extended chain length of 7 residue peptides in a $\beta$-sheet is $2.45 \mathrm{~nm}$. The radius of gyration of PEG with $\bar{M}_{\mathrm{n}}=3300 \mathrm{~g} \cdot \mathrm{mol}^{-1}$ is $1.98 \mathrm{~nm}$. For KLVFF-PEG the $\beta$-strand peptide length is $1.75 \mathrm{~nm}$, which together with the PEG chain dimensions yields a total domain spacing also in agreement with the opposed parallel $\beta$-sheet model in Figure 4.

Figure 3 shows SAXS profiles at room temperature, that is under conditions for which PEG is crystalline. The KLVFF-PEG sample shows a clear Bragg peak, consistent with the formation of well-defined lamellae. However, Bragg peaks are not observed for samples FFKLVFF-PEG and AAKLVFF-PEG. There is a shoulder of scattering at low $q$. This indicates that well-defined lamellae are not formed by these samples. This is consistent with observations of the morphology by polarized optical microscopy spherulites were observed for KLVFF-PEG but not

Table 1. Domain spacings in the melt $\left(70^{\circ} \mathrm{C}\right)$ and solid state $\left(20^{\circ} \mathrm{C}\right)$.

\begin{tabular}{lcc}
\hline Sample & $\frac{d\left(\mathbf{7 0}{ }^{\circ} \mathrm{C}\right)}{\mathrm{nm}}$ & $\frac{\boldsymbol{d}\left(\mathbf{2 0}{ }^{\circ} \mathrm{C}\right)}{\mathbf{n m}}$ \\
\cline { 2 - 3 } & 5.8 & 17.6 \\
KLVFF-PEG & 6.9 & - \\
FFKLVFF-PEG & 6.9 & -
\end{tabular}




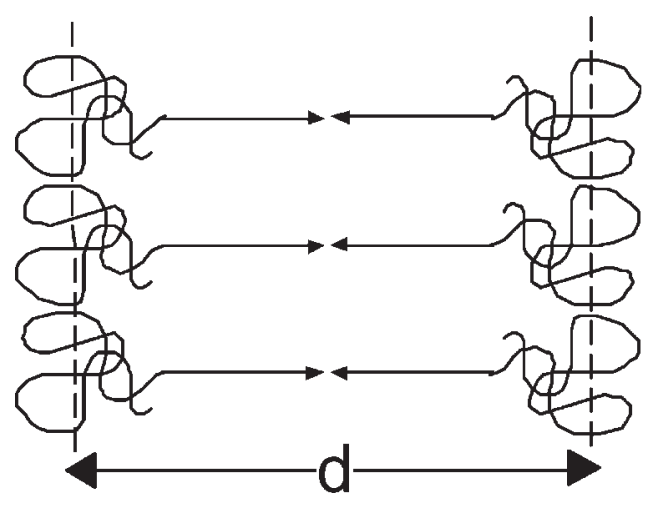

Figure 4. Opposed parallel $\beta$-sheet model for microphaseseparated structures in the melt.

FFKLVFF-PEG or AAKLVFF-PEG (Figure 5). The PEG is certainly crystallized in these samples, as confirmed by the presence of WAXS peaks (see for example Figure 1). The $d$ spacing observed for KLVFF-PEG provides evidence for chain folding of PEG. The extended chain length of PEG with $\bar{M}_{\mathrm{n}}=3300$ is $21.8 \mathrm{~nm}$ and with the length of $\mathrm{KLVFF}=1.8 \mathrm{~nm}$, the total length is $23.6 \mathrm{~nm}$. Since this is slightly larger than the observed $d$ spacing $(17.6 \mathrm{~nm})$ there may be interdigitation of polymer chains, or partial chain folding of the PEG. It appears however to be non-integral chain folding.

We also performed experiments in real space to image the morphology on different length scales. The macroscopic superstructure was revealed by polarized optical microscopy. Representative images are shown in Figure 5. The image for KLVFF-PEG in Figure 5a shows a characteristic spherulite structure from PEG crystallization. In contrast, Figure $5 b$ and Figure $5 c$ for AAKLVFF-PEG and FFKLVFF-PEG respectively do not exhibit a spherulitic structure. There is birefringence from an ill-defined liquid-crystal texture. These results correlate well with the presence or absence of a Bragg peak in the SAXS patterns (Figure 3).

AFM was used to probe the morphology at shorter length scales. Figure 6a shows fibrils for FFKLVFF-PEG. The twisted nature of the fibrils is evident. The height $(H)$ and width $(W)$ were estimated to be $H=(4.6 \pm 0.9) \mathrm{nm}$ and $W=(82.7 \pm 22.6) \mathrm{nm}$ (average over 10 fibrils), respectively. The fibrils formed by the PEG/peptide conjugate can be compared to the fibrils formed by the FFKLVFF peptide as shown in Figure $6 \mathrm{~b}$. The latter image was obtained for a more-concentrated solution $(0.25 \%$ in methanol compared to $0.0005 \%$ in methanol for the image in Figure $6 a$ ), hence the more-dense nature of the fibril packing. The height and width of the peptide were also measured, $H=(39.2 \pm 17.7)$ $\mathrm{nm}$ and $W=(56.6 \pm 10.8) \mathrm{nm}$ (average over 10 fibrils). The fibrils for the PEG conjugate are somewhat broader and have more diffuse boundaries, as expected due to the a)

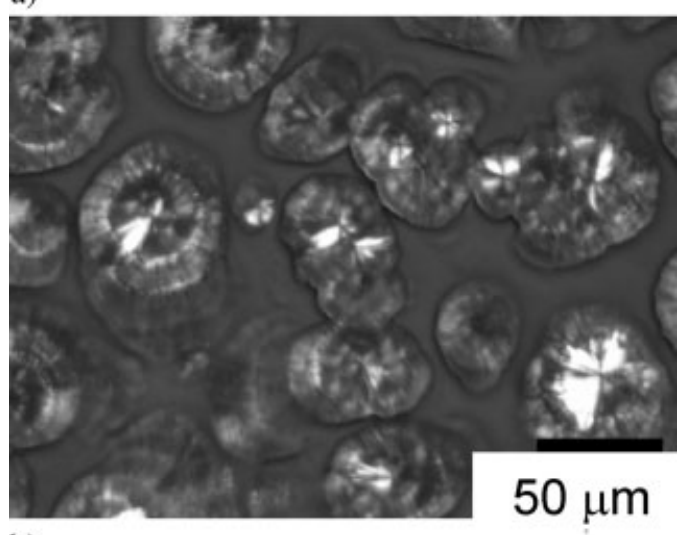

b)

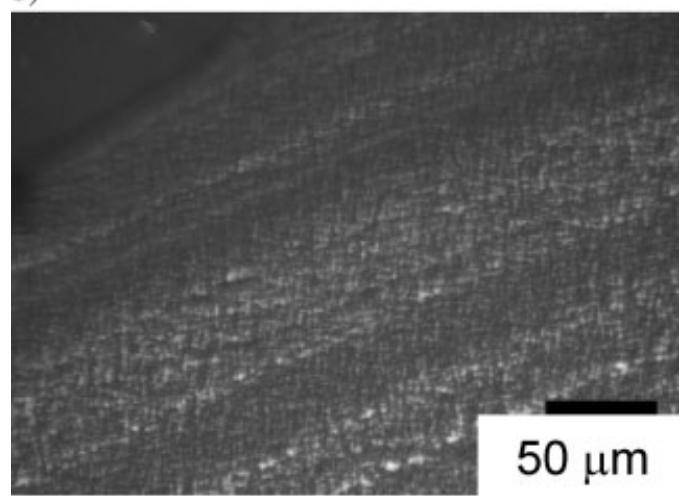

c)

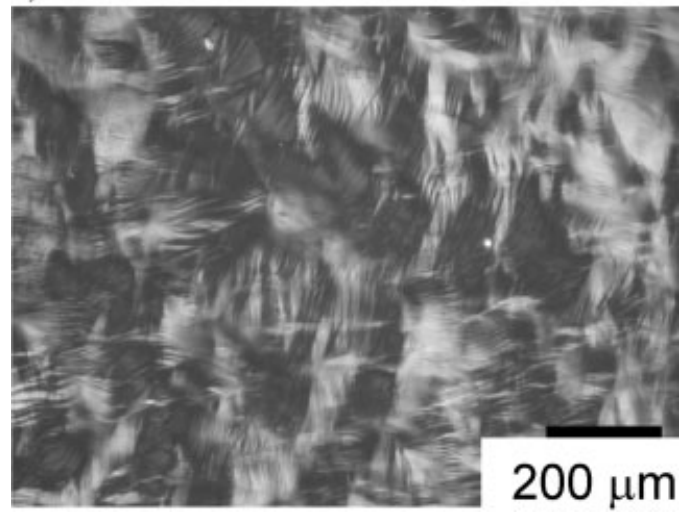

Figure 5. POM from dried films of (a) KLVFF-PEG, (b) AAKLVFF-PEG, (c) FFKLVFF-PEG.

presence of a PEG corona. The height of the PEGylated peptide is, however, significantly lower, which may reflect a drying artefact. In contrast to the fibrillar structure of FFKLVFF-PEG, AFM reveals a spherical domain structure for KLVFF-PEG (Figure 7). The diameters of the KLVFF-PEG aggregates were determined to be $H=(1.2 \pm 0.1) \mathrm{nm}$ and $W=(67.1 \pm 11.1) \mathrm{nm}$ (average over 10 spherical structures). The size of the aggregates is much smaller than those revealed by POM (Figure 5a). This may reflect the preparation conditions, since the AFM image was obtained 
a)

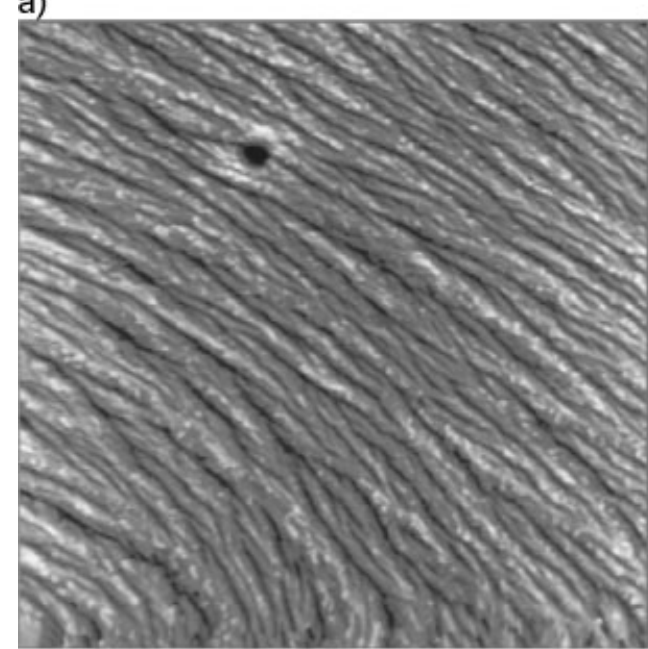

b)

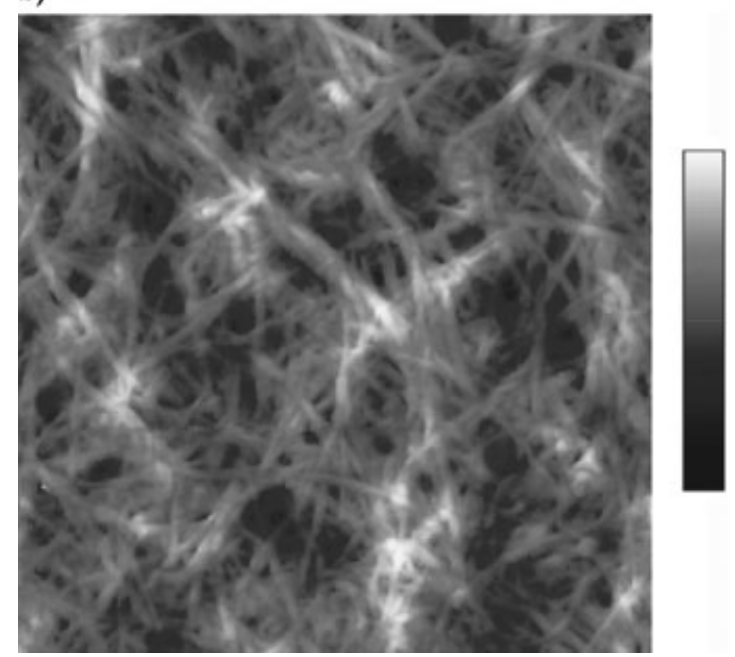

Figure 6. AFM height images for (a) FFKLVFF-PEG, prepared from 0.0005 wt.- $\%$ solution in methanol (the scan size of the image is $5 \times 5 \mu \mathrm{m}^{2}$ and the height scale is $0-14 \mathrm{~nm}$ ); (b) FFKLVFF, prepared from 0.25 wt.-\% solution in methanol (the scan size of the image is $5 \times 5 \mu \mathrm{m}^{2}$ and the height scale is $0-200 \mathrm{~nm}$ ).

after drying a very-dilute solution (0.0005 wt.-\% in methanol), which may prohibit the formation of large spherulites, and may only lead to the formation of initial crystal nuclei.

The FTIR spectra for FFKLVFF and FFKLVFF-PEG are compared in Figure 8. Similar spectra were obtained for the other samples, peak assignments being listed in Table 2 . The main focus of the FTIR experiments was on the amide I band, which is sensitive to secondary structure. Additional peaks to determine the peptide secondary structures were located in the amide A, amide B or amide II bands (Table 2). The PEG spectrum shows major bands in the vicinity of 950, 1100,1299 , and $1350 \mathrm{~cm}^{-1}$ which were associated with typical semicrystalline PEG (Table 2). ${ }^{[22]}$ In the amide

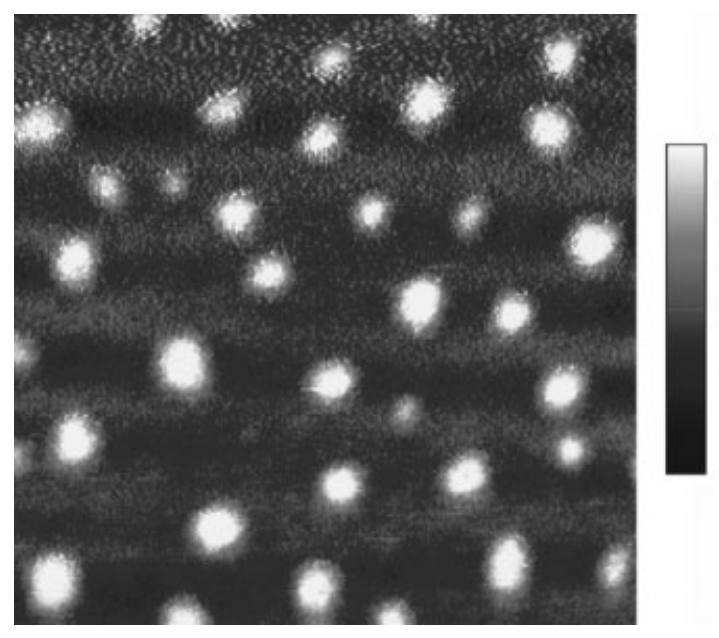

Figure 7. AFM height image for KLVFF-PEG prepared from a 0.0005 wt.-\% sample in methanol. The scan size of the image is $1 \times 1 \mu \mathrm{m}^{2}$ and the height scale is $0-1.4 \mathrm{~nm}$.

I region, the peaks at 1623 to $1629 \mathrm{~cm}^{-1}$ are certainly associated with a $\beta$-sheet structure. ${ }^{[23-27]}$ Peaks at 1673 to $1685 \mathrm{~cm}^{-1}$ as also observed for our samples, are often associated with an antiparallel $\beta$-sheet structure, ${ }^{[27]}$ although there is controversy in the literature on this. ${ }^{[28]}$ For this reason, FTIR spectroscopy cannot be used to unambiguously support our model (Figure 4) for opposed parallel $\beta$-sheets, although it is not inconsistent with this. Compared to the non-PEGylated peptides, the secondary structure of peptides was not significantly influenced by the PEG for FFKLVFF-PEG, although for KLVFF-PEG the amide I peaks were less intense (relative to the peaks assigned to semicrystalline PEG). However, there were distinct features resulting from PEG crystallization. As listed in Table 2, KLVFF-PEG has peaks at $1359 \mathrm{~cm}^{-1}$ and $1343 \mathrm{~cm}^{-1}$ while the other PEGylated samples have

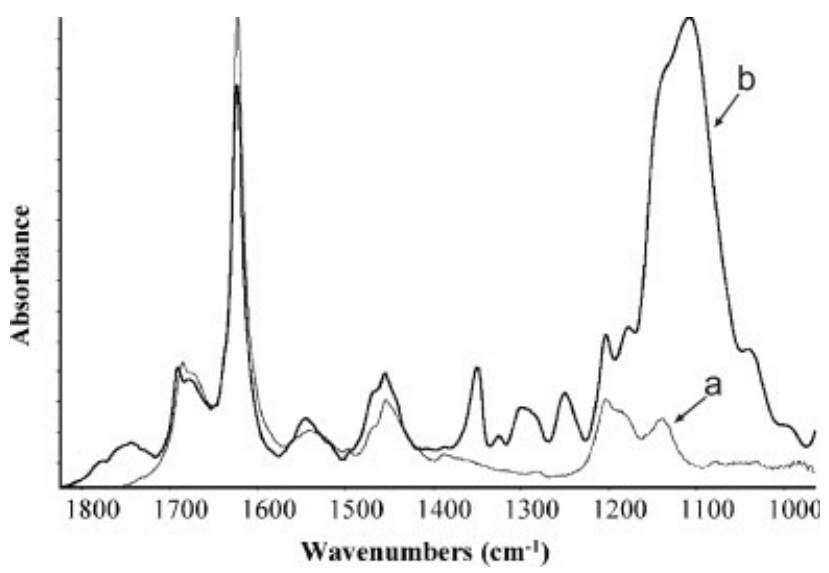

Figure 8. Representative FTIR spectra from (a) FFKLVFF and (b) FFKLVFF-PEG. 
Table 2. FTIR spectroscopy peak assignments.

\begin{tabular}{|c|c|c|c|c|c|}
\hline \multirow[t]{3}{*}{ Sample } & \multicolumn{5}{|c|}{ Peak assignments for different bands } \\
\hline & Amide $\mathbf{A}$ & Amide B & Amide I & Amide II & PEG \\
\hline & $\mathrm{cm}^{-1}$ & $\mathrm{~cm}^{-1}$ & $\mathrm{~cm}^{-1}$ & $\mathrm{~cm}^{-1}$ & $\mathrm{~cm}^{-1}$ \\
\hline KLVFF & 3274 & 3089 & 1673,1629 & 1554,1455 & - \\
\hline KLVFF-PEG & 3277 & 3088 & 1682,1626 & 1557,1467 & $1359,1343,1280,1242,963$ \\
\hline AAKLVFF & 3279 & 3031,3064 & 1668,1627 & 1557,1440 & - \\
\hline AAKLVFF-PEG & 3277 & 3089 & 1685,1625 & 1554,1455 & $1351,1325,1299,950$ \\
\hline FFKLVFF & 3275 & 3087 & 1685,1623 & 1544,1457 & - \\
\hline FFKLVFF-PEG & 3270 & 3087 & 1691,1623 & 1545,1455 & $1349,1325,1299,949$ \\
\hline
\end{tabular}

peaks at $1351 \mathrm{~cm}^{-1}$ (AAKLVFF-PEG) and $1349 \mathrm{~cm}^{-1}$ (FFKLVFF-PEG).

The broad absorption at approximately $2880 \mathrm{~cm}^{-1}$ was due to the $\mathrm{CH}_{2}$ asymmetric stretching bands and the $\mathrm{CH}_{3}$ symmetric stretching bands from the peptide. The peak was much more intense and broader for PEGylated samples which suggests an overlap with vibrations from the PEG. The CN and CO stretching bands correspond to peaks at 1202 to $1207 \mathrm{~cm}^{-1}$ which overlap with the vibration at 1105 to $1111 \mathrm{~cm}^{-1}$ from PEG (C-O-C stretching).

\section{Conclusion}

In summary, microphase separation is observed in the melt by SAXS. The observed Bragg-peak spacing corresponds to an opposed parallel $\beta$-strand configuration with extended $\beta$-strands and coil-like PEG. Upon crystallization, PEG adopts a partially-folded conformation and forms well-defined lamellae for KLVFF-PEG but not FFKLVFF-PEG or AAKLVFF-PEG. This is interpreted in terms of fibrillization capacity, which is enhanced in the latter two peptides due to the presence of additional hydrophobic residues; especially the additional phenylalanine units in FFKLVFF-PEG are predicted to enhance fibril formation. This is confirmed by computer analysis of sequences from protein databases. ${ }^{[29,30]}$ Spherulite formation by KLVFF-PEG is confirmed by POM and smaller aggregates presumed to be crystal nuclei are observed by AFM. In contrast, broad twisted fibrils are observed for FFKLVFF-PEG. The formation of fibrils by FFKLVFF-PEG prevents PEG crystallizing in lamellae due to the steric constraints imposed by the PEG attached to FFKLVFF fibrils.

Acknowledgements: We are grateful to Gemma Newby for assistance with the SAXS/WAXS experiments at HASYLAB.
Received: December 1, 2007 Revised: January 18, 2008; Accepted: January 22, 2008; DOI: 10.1002/macp.200700605

Keywords: block copolymers; microstructure; peptides; smallangle X-ray scattering (SAXS)

[1] G. W. M. Vandermeulen, H.-A. Klok, Macromol. Biosci. 2004, 4, 383.

[2] H.-A. Klok, J. Polym. Sci., Part A: Polym. Chem. 2004, 43, 1.

[3] D. W. P. M. Löwik, L. Ayres, J. M. Smeenk, J. C. M. van Hest, Adv. Polym. Sci. 2006, 202, 19.

[4] J. C. M. Van Hest, Polym. Rev. 2007, 47, 63.

[5] H. G. Börner, H. Schlaad, Soft Matter 2007, 3, 394.

[6] I. W. Hamley, Angew. Chem. Int. Ed. Engl. 2007, 46, 8128.

[7] D. B. Teplow, Amyloid 1998, 5, 121.

[8] M. Goedert, M. G. Spillantini, Science 2006, 314, 777.

[9] E. D. Roberson, L. Mucke, Science 2006, 314, 781.

[10] J. D. Sipe, Annu. Rev. Biochem. 1992, 61, 947.

[11] B. Caughey, P. T. Lansbury, Annu. Rev. Neuroscience 2003, 26, 267.

[12] H.-A. Klok, S. Lecommandoux, Adv. Polym. Sci. 2006, 202, 75.

[13] A. Rösler, H.-A. Klok, I. W. Hamley, V. Castelletto, O. O. Mykhaylyk, Biomacromolecules 2003, 4, 859.

[14] J. M. Smeenk, P. Schön, M. B. J. Otten, S. Speller, H. G. Stunnenberg, J. C. M. van Hest, Macromolecules 2006, 39, 2989.

[15] T. S. Burkoth, T. L. S. Benzinger, D. N. M. Jones, K. Hallenga, S. C. Meredith, D. G. Lynn, J. Am. Chem. Soc. 1998, 120, 7655.

[16] T. S. Burkoth, T. L. S. Benzinger, V. Urban, D. G. Lynn, S. C. Meredith, P. Thiyagarajan, J. Am. Chem. Soc. 1999, 121, 7429.

[17] P. Thiyagarajan, T. S. Burkoth, V. Urban, S. Seifert, T. L. S. Benzinger, D. M. Morgan, D. Gordon, S. C. Meredith, D. G. Lynn, J. Appl. Crystallogr. 2000, 33, 535.

[18] L. O. Tjernberg, D. J. E. Callaway, A. Tjernberg, S. Hahne, C. Lilliehöök, L. Terenius, J. Thyberg, C. Nordstedt, J. Biol. Chem. 1999, 274, 12619. 
[19] D. J. Gordon, R. Tappe, S. C. Meredith, J. Peptide Res. 2002, 60 37.

[20] M. J. Krysmann, V. Castelletto, A. Kelarakis, I. W. Hamley, R. A. Hule, D. J. Pochan, Biochemistry 2008, in press.

[21] I. W. Hamley, M. J. Krysmann, Biomacromolecules 2008, submitted.

[22] Y. Zheng, M. L. Bruening, G. L. Baker, Macromolecules 2007, 40, 8212.

[23] H. Susi, D. M. Byler, Arch. Biochem. Biophys. 1987, 258, 465.

[24] P. Haris, D. Chapman, Biopolymers 1995, 37, 251.
[25] B. Stuart, "Biological Applications of Infrared Spectroscopy", Wiley, Chichester 1997.

[26] M. Jackson, H. H. Mantsch, Crit. Rev. Biochem. Mol. Biol. 1995, 30, 95.

[27] J. T. Pelton, L. R. McLean, Anal. Biochem. 2000, 277, 167.

[28] A. Barth, C. Zscherp, Quart. Rev. Biophys. 2002, 35, 369.

[29] E. G. Hutchinson, R. B. Sessions, J. M. Thornton, D. N. Woolfson, Protein Sci. 1998, 7, 2287.

[30] A. P. Pawar, K. F. DuBay, J. Zurdo, F. Chiti, M. Vendruscolo, C. M. Dobson, J. Mol. Biol. 2005, 350, 379. 